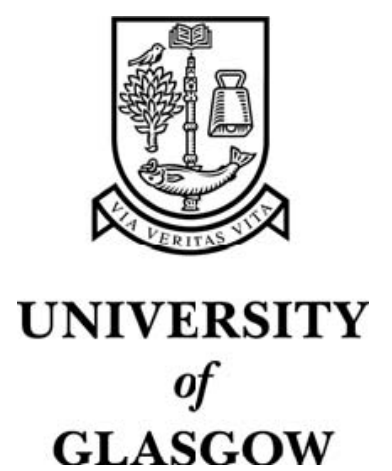

Xiao, Y. and Werghi, N. and Siebert, J.P. (2003) A topological approach for segmenting human body shape. In, 12th International Conference on Image Analysis and Processing, 17-19 September 2003, pages pp. 82-87, Mantova, Italy.

http://eprints.gla.ac.uk/3469/ 


\title{
A Topological Approach for Segmenting Human Body Shape
}

\author{
Yijun Xiao $^{1}$, Naoufel Werghi ${ }^{2}$, Paul Siebert ${ }^{3}$ \\ $(1,3)$ 3D-MATIC Lab, Department of Computing Science, \\ (2) College of Information Technology \\ University of Glasgow, UK \\ \{yjxiao,psiebert\}@dcs.gla.ac.uk \\ Dubai University College, UAE \\ nwerghi@duc.ac.ae
}

\begin{abstract}
Segmentation of $3 D$ human body is a very challenging problem in applications exploiting human scan data. To tackle this problem, this paper proposes a topological approach based on the Discrete Reeb Graph (DRG) which is an extension of the classical Reeb Graph to handle unorganized clouds of $3 D$ points. The essence of the approach concerns detecting critical nodes in the $D R G$ thereby permitting the extraction of branches that represent parts of the body. Because the human body shape representation is built upon global topological features that are preserved so long as the whole structure of human body does not change, our approach is quite robust against noise, holes, irregular sampling, frame change and posture variation. Experimental results performed on real scan data demonstrate the validity of our method.
\end{abstract}

Key words: 3D Segmentation, Human Body Shape, Discrete Reeb Graph, Critical nodes, surface anatomy

\section{Introduction and previous work}

The recent years have seen the emergence of 3D imaging technology that enables full scanning of the HB surface with reasonable measurement accuracy and acceptable computational cost. This advance facilitates the exploitation of the HB form in various areas such as anthropometrical research [1,2], clothing design $[3,4]$ and virtual human animation [5]. Although the raw data delivered by the HB scanner requires substantial main memory and backing store resources, this data contains little semantic information. For the effective and efficient use of body scan data, it is usually necessary to partition the whole scan data set into subsets corresponding to the different principal body parts. This segmentation provides the basis for a high-level representation of the scan data and is a prerequisite for further semantic analysis.

For example, in medical applications, the segmentation process provides an atlas for extracting data belonging to limbs that can be used to guide further analysis such as fitting generic limb models. These models can then be used to automate specific clinical protocols, such as spinal curvature assessment. Automatic segmentation of HB data is a challenging problem firstly because the body shape is both articulated and deformable and secondly because the scan data by nature is nonuniformly sampled, often containing gaps and corrupted by measurement noise.

The automatic segmentation of the HB scan data into the functional parts was pioneered by Nurre [6]. He approximated the body structure by a stick-template representing the head, the two arms, the two legs and the torso. His goal was to segment the body into six segments corresponding to these parts. This approach combines a global shape description, namely moments analysis, and local criteria of proximity which are derived from a priori knowledge of the relative positions of the body parts in the standard posture (standing body with arms held at the sides). The range data is organized into slices of data points. The horizontal slices are stacked vertically and the data points are assigned to the different body parts according to the slice's topology and its position in the body, e.g. a slice having two separated closed curves must represent data points measured at the level of the legs. A slice consisting of three closed curves must belong to the torso and arms area, a slice with two joined closed curves is assumed to correspond to the transition between the legs and the torso (at the level of the groin). Certainly this work illustrated a considerable progress towards the automatic decomposition of the HB data, however the approach is restricted to a strict standard posture and did not show evidence of robustness with respect to noise, gaps in the data, and variation in the shape and the posture of the HB. Using the framework of Nurre, Ju et al [7] refined the segmentation approach by introducing curvature analysis of profiles extracted from the slices to allow further decomposition of the body limbs into their articulated segments. Decker and Douros et al $[8,9]$ advanced Nurre's work by improving the localization of the key landmarks of the HB. For instance, they differentiated the slice circumferences in the torso area to locate the waist position. However their approach had the same limitations of Nurre's approach [6]. Recently, Wang et al [10] proposed an approach developed within a Fuzzy logic framework. As with Nurre's approach, a strict standing posture is assumed. After many preparation stages, the data is meshed and a 
segmentation technique applied that involves local curvature analysis of the slice data. However the overall performance of this approach remains identical to that of Nurre's.

In conclusion, the approaches developed to date are restricted by their underlying assumptions and may well suffer from instability when applied to real applications that must process noisy and corrupted 3D HB scan data containing posture variations. Furthermore, no evidence of the repeatability of these previous algorithms has been reported in the literature. For a HB data segmentation to be of practical utility, it must be robust to the variations of the body surface shape stemming from biological factors such as age, genetics, etc. It must also cope with reasonable perturbations in body posture. Adhoc techniques cannot satisfy these requirements, although they might work for a particular case. This paper proposes an approach defined within a general topological analysis framework. A systematic way to segment HB body data is presented that can cope with body shape variations and moderate posture changes. The approach does not require any pre-processing stages, operates on raw 3D point-cloud data and does not involve local feature analysis, which would be vulnerable to deficiencies in the scan data.

The rest of the paper is organized as follows: Section 2 describes the theoretical foundation of the approach and its relation with Morse theory and the Reed Graph. Section 3 describes the segmentation algorithm. The experiments and their results are presented in Section 4. The paper concludes in section 5 with a discussion of the results and future work.

\section{Morse Theory and Reeb Graph}

Morse theory can be thought of a as a generalization of the classical theory of critical points (maxima, minima and saddle points) of smooth functions on Euclidean spaces. Morse theory states that for a generic function defined on a closed compact manifold (e.g. a closed surface)) the nature of its critical points determines the topology of the manifold. Morse functions are generic functions for which all the critical points are nondegenerate (the Hessian matrix of the function at the critical point is non singular). For a Morse function, the critical points determine the homology groups of the manifold, that is a sets of points for which the function is less than a given value $x$. Moreover these sets can fully describe the topology of the manifold. The way the manifold is embedded in the 3D space can be coded using the Reeb graph which is a skeleton graph that encodes the evolution and the arrangement of the homology groups.
Reeb graph represents the configuration of critical points and their relationship and provides a way to understand the intrinsic topological structure of a shape. Consequently, the Reeb graph has been used in many applications such as shape matching [11], shape coding [12] and surface description and compression [13, 14]. A Reeb graph is defined as follows:

Definition 1 (Reeb graph): Let f be a real-valued function on a compact manifold $M$. The Reeb graph of a function $f$ is the quotient space of the graph of $\boldsymbol{f}$ in $M$ by the equivalence relation “ ", given by:

$(X 1, f(X 1)) \sim(X 2, f(X 2))$ iff $f(X 1)=f(X 2)$ and $X 1$ and $X 2$ are in the same connected component of $f^{-1}\left(f\left(x_{1}\right)\right)$

Roughly speaking, the two points $(X 1, f(X 1))$ and $(X 2$ $, f(X 2))$ are represented as the same element in the Reeb graph if the values of $f$ are the same and if they belong to the same connected component of the inverse image of $f(X 1)$ (or $f(X 2)$ ). Actually one element in the Reeb graph of a compact manifold represents all points having the same value under a real function. Figure. 1 illustrates an example of Reeb graph for a torus (a). The critical points associated to the chosen 'height' function are depicted. The corresponding Reeb graph is shown in (b) where triangular nodes denote local extremal points and square nodes denote saddle points.

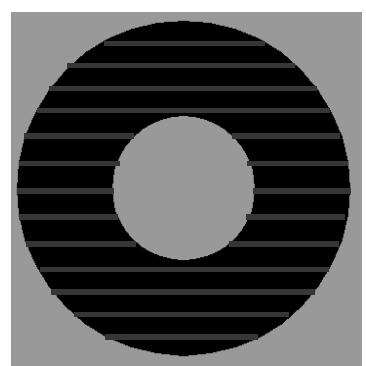

(a)

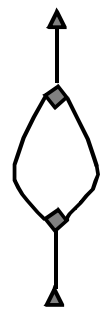

(b)
Figure 1: Reeb Graph of a Torus

By applying Reeb Graph to a human figure, we can get a skeletal representation as illustrated in Figure 2, where the height function is adopted. It can be seen that the critical points have important meanings on the skeletal figure. Extremal points represent head top, hand tips and foot toes. Saddle points represent armpits and groin. Moreover, the branches in the graph reflect the body parts of the figure, i.e., arms, legs, torso and head. Therefore, if we collect the level-set curves in these branches, we can obtain the data corresponding approximately to the body parts of the human figure. This is the key idea behind our proposed approach.

\subsection{Discrete Reeb Graph}


The classical Morse theory is concerned with only nondegenerate critical points of smooth functions (Morse function) on smooth manifolds. In practice, our data format does not comply with this restrict assumption, as it consists of points sampled on the measured human body surface, and it might be corrupted by noise and gaps. The construction of the Reeb-Graph for such data, is inspired from the approach of Biasotti et al $[13,14]$ who proposed an Extended Reeb Graph which can be extracted from a discrete surface, where the data is described by a set of unorganized polygonal contours. In our case the data is an unorganized cloud of $3 \mathrm{D}$ data points. We call the Reeb graph extracted from such data the Discrete Reeb Graph (DRG).

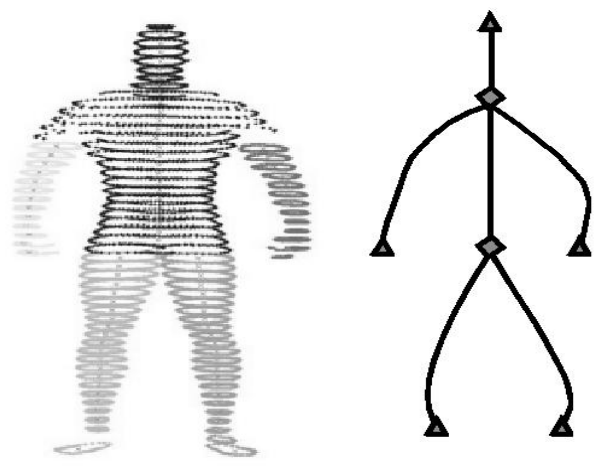

Figure.2: Reeb graph of a human figure.

The extraction is based on the notion of connectivity described in the following definitions:

\section{Definition 2 (connectivity of point sets):}

Two point sets $P=\left\{p_{i}\right\}, i=1 . . m$ and $Q=\left\{q_{j}\right\}, j=1 . . n$ are defined as connected iff

$\exists p_{i} \in P \quad$ and $\quad q_{j} \in Q$ such that $\left|p_{i}-q_{j}\right| \leq d$ Where

$\left|p_{i}-q_{j}\right|$ means the distance between points $p_{i}$ and $q_{j}$ and $d$ is a threshold that denotes the maximum distance between a couple of 'connected' points. The above definition covers also the connectivity between two points for the particular case where the sets $P$ and $Q$ each contain a single point.

\section{Definition 3 (connective point set):}

$A$ point set $C$ is connective iff

$\forall \Omega \subset C$ and $\Omega \neq \phi, \Omega$ and $\bar{\Omega}$ are connected,

where $\bar{\Omega}$ is the complement set of $\Omega$ in $C$, and $\phi$

denotes the empty set.

Based on the above definitions the DRG is built on the discrete set of points according to the following steps:

Step1: Establishing level-set curves

For a continuous surface, a level-set curve is the intersection between a plane with a certain height and the surface. Due to the discrete nature of the data (point samples), we extract data in a slice with a height in the domain $[h, h+d]$, where $h$ is a height value and $d$ is the slice thickness. By choosing an appropriate value of $d$, each slice will contain a sufficient number of data points for analysis. The data is sliced from bottom to top. In total there are ceil $\left(h_{\max }-h_{\min } \mid / d\right)$ slices. Here ceil (.) represents the nearest integer towards positive infinity and $h_{\max }, h_{\min }$ denote the maximum and minimum height value of the data. For each slice, data points are grouped into several discrete connective sets consisting of connected data points based on the definition of connectivity above. These groups which we call discrete curves represent the level-set curves in the DRG.

Step2: Building the connectivity graph

Considering each curve as a node in the graph, two nodes in two adjacent slices respectively are linked if their corresponding curves are connected. In this way, we can build up a graph containing all nodes and their links, i.e., DRG.

\section{Note}

In the DRG, critical points might degenerate into 'critical curves'. In other word, we might not discover the exact critical points due to the discreteness of data and slicing process, instead these points appear to be critical curves around which the topology of human body varies. Fortunately, our application does not deal with the theories based on non-generate critical points that many other applications involve. Therefore we do not distinguish critical points and critical curves in our application, and generally we title them 'critical nodes' in the DRG.

\section{Segmentation Algorithm}

Before presenting the details of the segmentation algorithm, we describe our problem in a precise manner. First of all, the subject of our study is a general human figure. The $\mathrm{HB}$ data can be acquired by a general $3 \mathrm{D}$ scanner that is not constrained by specific specification requirements. The data is corrupted with noise, holes and gaps. Moreover, the human figure stands in the measuring platform with arms held at the sides and legs separated. Therefore the height direction of the measured figure is known. In order to segment scan data according to DRG, we need firstly to extract critical nodes in the DRG, and then locate branches representing body parts, finally we retrieve the data points corresponding to the branches. Finding critical nodes in the classical Reeb Graph is very intuitive. The root and leave nodes represents local extremal points. The branched nodes represent saddle points. However, it is nontrivial to extract critical nodes in $\mathrm{DRG}$, because the noise and holes in the data might 
change the local topological properties of the scan data and create 'false' critical nodes. In order to detect the 'true' critical nodes, we assume that the effects of noise and holes on the topology are bounded. Thus, we can use the following criteria to identify 'true' critical nodes

- A 'true' saddle node has at least two 'true' branches all of which are longer than a threshold length.

- 'True' extremal nodes are the deepest leaves of 'true' branches.

The segmentation algorithm is based on the analysis of the DRG and the above criteria. The algorithm contains only one pass searching from the bottom to the top of data. In this pass, the critical nodes representing foot toes, groin, hand tips, armpits and head top are detected using the criteria mentioned above and the 'true' branches between these critical nodes are extracted. Then the identification of branches corresponding to the body parts becomes very simple. The branches between groin and foot toes correspond to legs. The branches between armpits and hand tips correspond to arms. The rest of the data correspond to torso and head. The algorithm for building the DRG and finding the 'true' saddle nodes and branches is described by the following pseudo-code

\section{Notation:}

Node - A node is an entity which contains a curve in a slice.

NewNode() - A function to allocate a new node

Class - A class is defined as a group of connected nodes.

Class(Node) - The Class containing the Node.

NewClass() - A function to allocate a new class

Num(Class) - The number of nodes in the class

Threshold - A number used in our criterion for detection of 'true' branches.

Branch - The 'true' branch connected to a 'true' saddle node.

\section{Code:}

Slicing from bottom to top

For each slice

Group data points into curves

For each curve

node: $=$ NewNode( $)$

If it is the 1-st slice

Else

$$
\text { Class (node) }:=N e w \operatorname{Class}()
$$

Determine the nodes $\left\{N_{1}, N_{2}, \ldots, N_{m}\right\}$ in last

slice connected to the node

If $m=0$

Class (node) $:=N e w C l a s s()$

\section{Else}

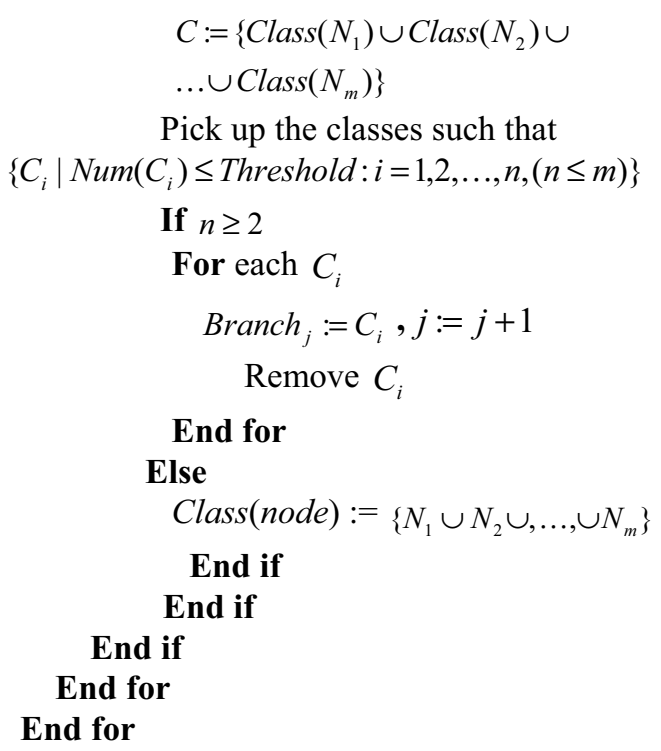

To simplify the curve grouping process in the above algorithm, we partition regularly the space of a slice into cubes each with the size of $d \times d \times d$. If there are data points inside a cube, this cube is labeled as ' 1 ', otherwise labeled as ' 0 ' (Fig.3). We can process these cubes according to their index in the same manner as a binary $2 \mathrm{D}$ image. Finding connected component in an image has been well examined in the literature. We choose a standard technique mentioned in [15](Chapter 2) to achieve this task.

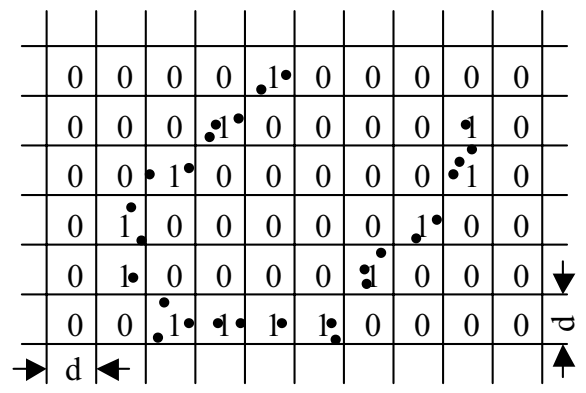

Figure 3 Finding connected curves in a slice

\section{Experiments}

The validity of our method was tested through experiments conducted on real data samples. The first group of samples was downloaded from Cyberware website [16]. The samples, acquired from Cyberware whole body 3D scanner-WB4, include two female and two male subjects. Each sample contains about 12,000 data points. Figure 4 shows the pictures of the subjects and the segmentation results of their $3 \mathrm{D}$ scan data. It can be noticed that the body joints at the level of the armpits and the groin were detected quite faithfully, and the 
segmented parts of the data correspond reasonably to the arms and legs.

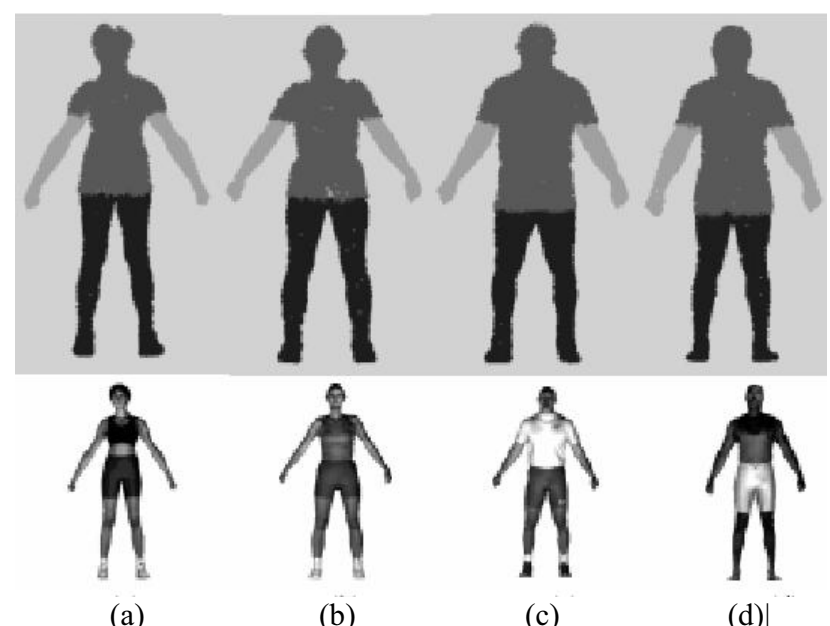

Figure 4: Segmentation of the Cyberware samples

It is also worth noting that the results were obtained using low quality scan data, as illustrated in Figure 5 which shows a zoomed area around the groin in Figure 4(a). The non-regular sampling of the data and the presence of gaps and holes can be observed. Hence, the algorithm appears to be robust to the above data corruption models.

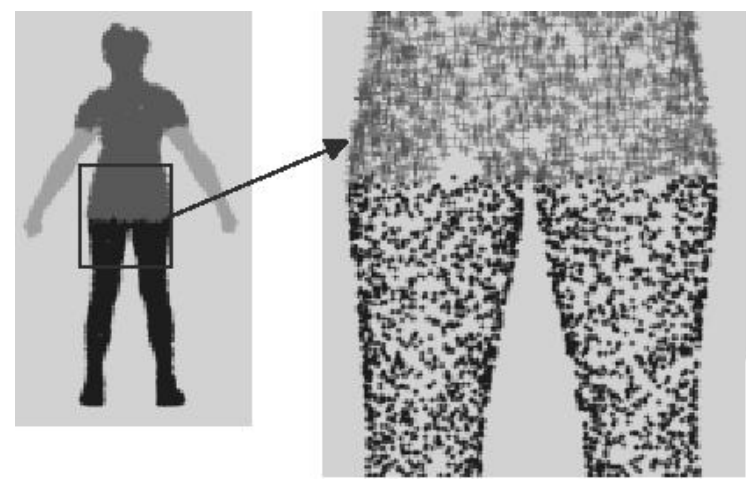

Figure 5: Zoomed image illustrating the distribution of the scan data.

The choice of the height function used in the construction of the DRG assumes that the principal orientation of the body is collinear to the $\mathrm{z}$ axis of the coordinate frame. However this assumption might not hold due to many reasons such as the presence of a systematic error in the scan data or variations in the body standing pose. The effects of violating the $\mathrm{z}$ axis colinearity assumption were simulated by perturbing the coordinate frame orientations. This was implemented by rotating the coordinate frame around its $\mathrm{x}$ and $\mathrm{y}$ axes. To check the robustness of the algorithm, the perturbation was pushed to an extreme case by setting the angle of rotation to $30^{\circ}$ and $-30^{\circ}$ (Figure. 6 (a)). The experiments were carried out on the body scan data of Figure 4(a) and the results are are displayed in Figure 6. The figure shows that the algorithm still produces a segmentation faithful to the body anatomy. However, the segmentation near the groin and the armpits is of reduced accuracy due to the difference between the slicing orientation and principal orientation of the body. Nevertheless these results indicate that the algorithm can cope (to a reasonable extent) with uncertainty in the principal orientation of the body.

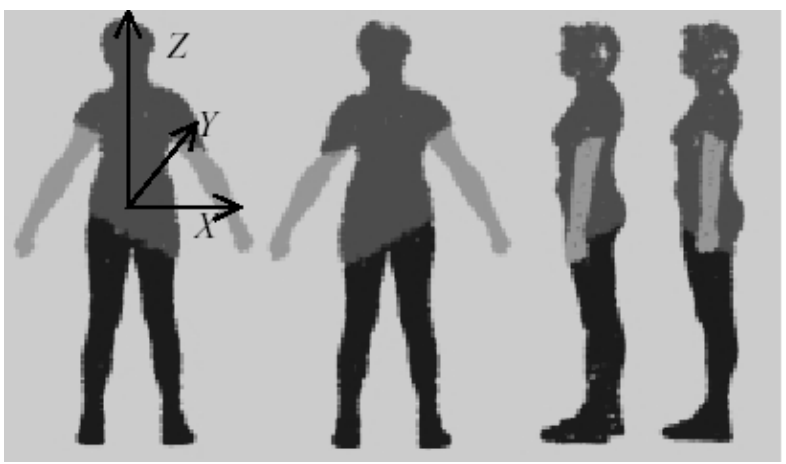

(a)

(b)

(c)

(d)

Figure 6: Segmentation results obtained from perturbed data. (a) and (b) data rotated around the y axis by $30^{\circ}$ and $-30^{\circ}$ respectively. (c) and (d) data rotated around the $\mathrm{x}$ axis by $30^{\circ}$ and $-30^{\circ}$ respectively.

The second group of experiments was performed on human body scans acquired from 3D human body scanner at the EDVEC center [16]. This scanner uses Moire' fringe based $3 \mathrm{D}$ imaging technology, and is thus quite different from the Cyberware scanner which uses Laser technology. The scans are related to one subject in three different postures (Figure 7(a)). Each scan contains about 11,000 data points. The algorithm worked in a satisfactory way resulting in a reasonably accurate segmentation for the three postures (Figure 7(b)).

\section{Conclusion}

This paper presents a new approach, based on topological analysis, for segmenting human body data scans. The approach extends the Reeb graph framework to the case of unorganized cloud of data points by defining and utilizing connectivity concepts. The approach can handle directly raw scan data without the need for any preprocessing or pre-formatting of the data. The segmentation proved to be robust against noise, gaps and irregular sampling and has no dependency on the specifications of the scanners. The performance of the algorithm was confirmed by experiments carried out with real scan data, acquired from different sources and related to a variety of human figures and postures. All the figures were successfully segmented into five parts without any manual intervention. To the best of our knowledge this 
work is the first to present a reliable and repeatable approach for segmenting 3D Human body scans.

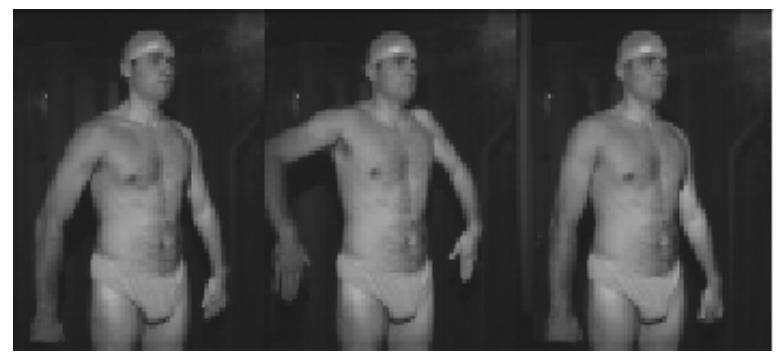

(a)

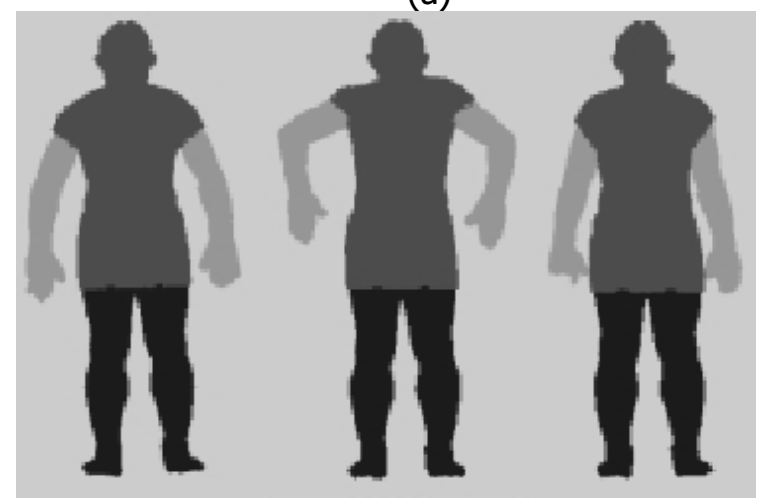

(b)

Figure 7: Three body postures (a) and their related segmented scans

Currently, the range of variation we allow in body posture is limited. Accordingly, we are investigating an extension to the presented algorithm to enable it to cater for a greater variety of body poses. This work is based on the concept of building multiple and complementary ReebGraphs constructed using appropriate generic functions. The analysis of each graph would then be combined to produce a single robust segmentation.

\section{References}

[1] P.R.M. Jones, M. Rioux, "Three dimensional surface anthropometry:applications to human body", Optics and Lasers in Engineering, Vol. 28, No. 2, pp.89- 117, 1997.

[2] E. Paquet, K.M Robinette, M. Rioux, "Management of three-dimensional and anthropometric databases: Alexandria and Cleopatra”. J. Electronic Imaging, Vol. 9, pp. 421-431, 2000.

[3] R.P. Pargas, N.J. Staples, J.S. Davis, "Automatic measurement extraction for apparel from a threedimensional body scan", Optics and Lasers in Engineering, Vol. 28, No. 2, pp.157-172, 1997.

[4] F. Cordier, H. Seo, N. Magnenat-Thalmann, "Madeto-MeasureTechnologies for an Online Clothing Store",
Computer Graphics and Applications, pp.38-48, (1) 2003.

[5] J. Starck, G. Collins, R. Smith, A. Hilton, and J. Illingworth, "Animated statues", Journal of Machine Vision Applications, 2002.

[6] J.H. Nurre, "Locating landmarks on human body scan data" Proc Conf. 3D Digital Imaging and Modeling, pp.289-295, Ottawa, Canada, 1997.

[7]X. Ju, N. Werghi, and J. P. Siebert, "Automatic Segmentation of 3D Human Body Scans", Proc. Int. Conf. on Computer Graphics and Imaging 2000 s Vegas, USA, 2000.

[8] L. Dekker I. Douros B. F. Buxton P. Treleaven, "Building Symbolic Information for 3D Human Body Modeling from Range Data", Proc. 3D Digital Imaging and Modeling, Conf. 1999, pp. 388-397, Ottawa, Canada.

[9] I. Douros, L. Dekker, B. Buxton, "Reconstruction of the surface of the human body from 3D scanner data using B-splines", Proc. SPIE vol. 3640, pp234-245, San Jose, California, January 1999.

[10] C.L Wang, T.K, Chang, M. Yuen, "From laserscanned to feature human model: a system based on fuzzy logic concepet”, CAD, Vol.35, pp. 241-253, 2003.

[11] M. Hilaga, Y. Shinagawa, T. Kohmura, T. .Kunii, "Topology matching for fully automatic similarity estimation of 3d shape". SIGGRAPH 2001, pp 203 212, New York, USA.

[12] Y. Shinagawa, T.L. Kunii and Y.L. Kergosien. "Surface Coding Based on Morse Theory" , IEEE Computer Graphics and Applications, Vol.11, No.5, pp.66-78, 1991.

[13] S. Biasotti, M. Mortara, M., M. Spagnuolo, "Surface Compression and Reconstruction using Reeb graphs and Shape Analysis, Proc. of Spring Conference on Computer Graphics, pp. 175-184, Bratislava 2000.

[14] S. Biasotti, B. Falcidieno, M. Spagnuolo, "Extended Reeb Graph for Surface Understanding and description" Baja (eds) Lectures notes in Computer Sciences, 1953, pp. 185-197, 2000

[15] R.M. Haralick, L.G. Shapiro, Computer and robot vision, Vol. 1, pp.33-37, Addison-Wesley, 1991.

[16] www.cyberware.com 
[17] Edinburgh Virtual Reality Center, www.edvec.ed.ac.uk 